\title{
Cultivating a Grassroots Aerospace Innovation Culture at NASA Ames Research Center
}

\author{
Sarah D'Souza, Hugo Sanchez, and Ryan Lewis \\ NASA Ames Research Center \\ Moffett, CA 94035
}

\begin{abstract}
This paper details the adaptation of specific 'knowledge production' methods to implement a first of its kind, grassroots event that provokes a cultural change in how the NASA Ames civil servant community engages in the creation and selection of innovative ideas. Historically, selection of innovative proposals at NASA Ames Research Center is done at the highest levels of management, isolating the views and perspectives of the larger civil servant community. Additionally, NASA innovation programs are typically open to technical organizations and do not engage non-technical organizations to bring forward innovative processes/business practices. Finally, collaboration on innovative ideas and associated solutions tend to be isolated to organizational silos. In this environment, not all Ames employees feel empowered to innovate and opportunities for employee collaboration are limited. In order to address these issues, the 'innovation contest' method was adapted to create the NASA Ames Innovation Fair, a unique, grassroots innovation opportunity for the civil servant community. The Innovation Fair consisted of a physical event with a virtual component. The physical event provided innovators the opportunity to collaborate and pitch their innovations to the NASA Ames community. The civil servant community then voted for the projects that they viewed as innovative and would contribute to NASA's core mission, making this event a truly grassroots effort. The Innovation Fair website provided a location for additional knowledge sharing, discussion, and voting. On March 3rd, 2016, the 'First Annual NASA Ames Innovation Fair' was held with 49 innovators and more than 300 participants collaborating and/or voting for the best innovations. Based on the voting results, seven projects were awarded seed funding for projects ranging from innovative cost models to innovations in aerospace technology. Surveys of both innovators and Fair participants show the Innovation Fair was successful in fostering cross-organizational collaborations, soliciting participation of non-technical innovations, and increasing employee engagement in influencing the future of NASA Ames Research Center. The grassroots component of the Innovation Fair has been bench marked by the agency as a solid foundation for increasing employee engagement in the development of game changing aerospace technology and processes in support of NASA's mission.
\end{abstract}

\section{TABle of Contents}

1. INTRODUCTION ............................

2. Challenges to InNovation at NASA $\ldots \ldots \ldots \ldots 2$

3. InNOVATION FAIR CONCEPT ................. 3

4. ReSUlts ................................ 5

5. LESSONS LEARNED $\ldots \ldots \ldots \ldots \ldots \ldots \ldots \ldots \ldots . \ldots . \ldots$

6. Conclusion $\ldots \ldots \ldots \ldots \ldots \ldots \ldots \ldots \ldots \ldots \ldots . \ldots . \ldots . \ldots$

APPENDICES ............................... 8

ACKNOWLEDGMENTS .........................9

REFERENCES $\ldots \ldots \ldots \ldots \ldots \ldots \ldots \ldots \ldots \ldots . \ldots \ldots$

BIOGRAPHY $\ldots \ldots \ldots \ldots \ldots \ldots \ldots \ldots \ldots \ldots \ldots \ldots \ldots . \ldots \ldots$

U.S. Government work not protected by U.S. copyright

\section{INTRODUCTION}

The aerospace industry is constantly working to address challenging problems, from traveling beyond Earth's orbit to revolutionizing aircraft propulsion technology. Some of these challenges are uniquely suited to NASA's mission and will require innovative technologies and processes, keeping NASA on the cutting edge of the aerospace frontier. Historically, innovations have been promoted at the highest levels of management, isolating the views and perspectives of the larger civil servant community. In this environment, not all employees feel empowered to innovate and opportunities for employee collaboration become limited. NASA Administrator, Charlie Bolden, recognized this when he stated to the NASA workforce, "I believe there is more that can be done to help every employee feel that they have the opportunity to be innovative. It is critical to NASA's identity that we constantly push the boundaries of what humans believe is possible." The objective of this innovation effort is to create this opportunity and break through old practices by: I) promoting an innovation culture in NASA's technical and non-technical organizations, II) providing broad collaborative opportunities that foster innovation across organizations, and III) increasing employee influence on NASA's future. Successfully achieving these objectives can cultivate a powerful culture of innovation within NASA.

This paper details the implementation of a first of its kind event at NASA Ames Research Center (ARC) aimed at cultivating this culture of innovation through the adaptation of 'knowledge production' methodologies [1], where knowledge production is the resulting outcome of the implementation and use of a particular methodology, e.g. traditional collaboration, crowd sourcing, etc. The goal is to design a grassroots opportunity for NASA Ames civil servants from all organizations to leverage peer support in order to receive seed funding and management recognition of innovative concepts. The definition of grassroots, in this context, is the use of civil servant voting results to select innovation concepts. Section 2 discusses the challenges to innovation at NASA, section 3 details the adaptation of innovation practices to create this opportunity for civil servant employees and the metrics used to measure success, section 4 documents the results of the Innovation Fair, and section 5 discusses lessons learned. Note that the term 'technical organizations' refers to exploration technology, aeronautics, engineering, information technology, and science groups, while 'non-technical organizations' refer to partnerships, finance, administration, human capital, procurement, safety, and education groups.

Finally, it should be noted that as the Innovation Fair occurred less than one year from the writing of this paper, long term data is not yet available for analysis. Therefore, the context and focus of this paper is on the data that has already been collected and short term indications of success as it relates to the objectives outlined above. Further research on a longer time horizon will be required to validate early findings and 
get a better understanding of the true impact of the Innovation Fair to ARC's innovation culture and mission success.

\section{Challenges to InNovation at NASA}

The authors were teamed by NASA ARC and asked to determine and implement measures that would have a high impact on the ARC work force. In order to identify high impact measures, the team set out to research current ARC activities and workplace climate through interviews, innovation benchmarks, and a literature review.

Interviews were conducted with NASA Agency senior management, ARC senior management, and ARC colleagues to understand where each saw the need for change. Discussions with senior management revealed that management is actively implementing methods to promote and maintain employee engagement in the health and future of NASA. Discussions with NASA ARC co-workers revealed that many employees have innovative ideas to improve NASA technology and processes, but there are barriers to an employee's ability to grow these ideas, adversely affecting employee engagement. The disconnect between what management is trying to achieve and the climate of the workforce led the team to research current programs that promote an innovation culture across the Agency and internally at ARC to determine the contributing factors to this disconnect.

In the Space Technology Directorate, there are a number of programs that solicit proposals for new ideas, namely, the Centennial Challenges [2], Center Innovation Fund [3], NASA Innovative Advanced Concepts [4], Space Technology Research Grants [5], Game Changing Development Program [6], and Technology Demonstration Missions [7]. In the Aeronautics Directorate proposals are requested under the NASA Aeronautics Research Institute [8] and the Transformative Aeronautics Concepts program [9]. Additionally, the Office of the Chief Scientist has an Innovation Fund. Each of these efforts, while important to each field's development, have several aspects in common that hinder an innovation culture. First, these programs are targeted to technical organizations and focused solely on technology development, isolating process improvements that could revolutionize how NASA does business. Second, significant proposal writing and idea development is typically required, a priori. New ideas have very little results or associated research, so most employees with new ideas cannot pursue these programs as a means to grow the innovations due to the proposal process. Finally, awards are made based on high-level senior panels, which sometimes promotes a perception that selection is biased toward those with the most senior level exposure.

Additionally, NASA's Office of the Chief Technologist published a white paper [11] that addressed the barriers to innovation and found several common themes that align with what the team found through interviews and current efforts. First, the use of management oversight bodies promote an overly risk averse culture and unnecessarily slow early innovation growth. Second, the process is overloaded with excessive administrative tasks, such as proposal writing, lengthy mid-term reports, meetings, etc. Third, the existence of organizational silos promote activities that isolate organizations and hinder broad collaboration.

In order for this effort to meet the objectives outlined in Section 1, the barriers generated from management oversight bodies, process overload, and organizational silos must be addressed. Thus, the team researched a variety of methodologies that promote knowledge production in the work place. These methods are separated into four categories [1]:

- Traditional Mertonian science [13] - Defined by a confined set of subject matter experts whose methods and data are largely kept in-house due to workplace culture placing an emphasis on gaining recognition for being the first to publish new research. In this case external participation and intermediate methods/data are closed.

- Traditional science with disclosures - Defined by a confined set of subject matter experts whose methods and data are published in the public domain upon completion of the work. In this case external participation is closed and intermediate methods/data are partially disclosed in the open.

- Innovation contests/crowd sourcing [14] - Defined by a diverse set of individuals contributors whose methods and data are not publicly disclosed due to project sponsors funding these efforts to seek a competitive advantage. In this case external participation is open and disclosure of intermediate methods/data are not open.

- Crowd science [15] - Defined by a diverse set of individual contributors whose methods and data are shared publicly throughout the course of development. This method has typically been successful in reducing solution times and cost for large scale problems, e.g big data, etc. In this case external participation and intermediate methods/data are fully open.

The traditional methods in the first two bullets exemplify the old practices that target specific technical organizations, require extensive proposal writing, and rely on high-level senior panels to award research funding. The methodology that uses innovation contests and crowd sourcing has the potential to meet Objectives I - III based on the diversity of participants that are called to contribute and collaborate on innovative ideas. The advantage of this method is in the competitiveness among a diverse set of participants resulting in a broad range of solutions. These efforts are largely completed on web-based platforms. The crowd science methodology has the potential to provide guidance for a full and open framework that addresses Objectives I - III. The advantage of this method is in the diverse set of participants and the transparency in optimal solutions/methods. These efforts have been completed on web-based platforms and at physical events (e.g. the Foldit game for protein folding [1], [16]).

Versions of these method have been implemented by NASA to address some of the barriers to innovation. For example, NASA's Information Technology (IT) Directorate implemented NASA IT Labs innovation, which is an innovation contest where the application for entry has been minimized to reduce process overload and awardees are selected via a panel. Additionally, NASA's Office of Human Capital Management released the Innovation Pavillion [10], which is a web-based platform that allow employees to virtually exchange ideas across the ten NASA centers, breaking down some of the organizational silos. At Marshall Space Flight Center, an Innovation Day "Shark Tank" was held where panel selection and audience voting determined which innovation to fund. Similarly, NASA's Kennedy Space Center and Johnson Space Center implemented an Innovation Expo and Innovation Day, respectively, to provide a venue for collaboration and conduct innovation contests where awardees are selected via a panel. Each of these programs maintain the role of a high-level panel selecting innovation awardees and/or does not provide a physical venue for cross-organizational 
collaboration.

Based on the lack of a grassroots innovation opportunity within NASA, where the civil servant community selects innovation for seed funding, the team embarked on the development and implementation of an Innovation Fair opportunity that adapts the innovation contest method to meet Objectives I - III. Due to government restrictions on methods/data release, adapting the crowd science model is not currently feasible. In addition, government restriction only allowed government civil servants to submit proposals and serve as Principal Investigators for the Innovation Fair.

\section{INNOVATION FAIR CONCEPT}

\section{Overview}

The primary objective of the Innovation Fair was to fund projects that lead to innovation and advance agency mission goals through collaboration. Many innovation projects across NASA have attempted similar efforts in the past, but the funding awards typically targeted specific technology development, rarely giving process improvements an opportunity to compete for funding. Before selection and funding, these innovative concepts also received limited exposure across the center, with only a handful of management or peers serving as concept reviewers.

Three unique aspects separate the Innovation Fair from previous 'innovation contest' efforts at NASA. First, the call is open to all organization divisions at ARC, technical and nontechnical. Second, the results of a democratic vote are used by management to award funds, making this a unique grassroots approach to recognizing potentially innovative ideas within NASA. Third, the Innovation Fair and associated website serve as an idea capture and collaboration forum in support of fostering cross-organizational collaborations.

The following subsections detail the virtual component, physical component, voting methodology, award methodology, and success metrics applied to the Innovation Fair.

\section{Virtual Component}

The Innovation Fair website served as the primary portal for general event information, proposal submission, innovation concept discussion, and voting. The website was built using an established Microsoft SharePoint [12] service that operates across the agency, as shown in Figure 1. The

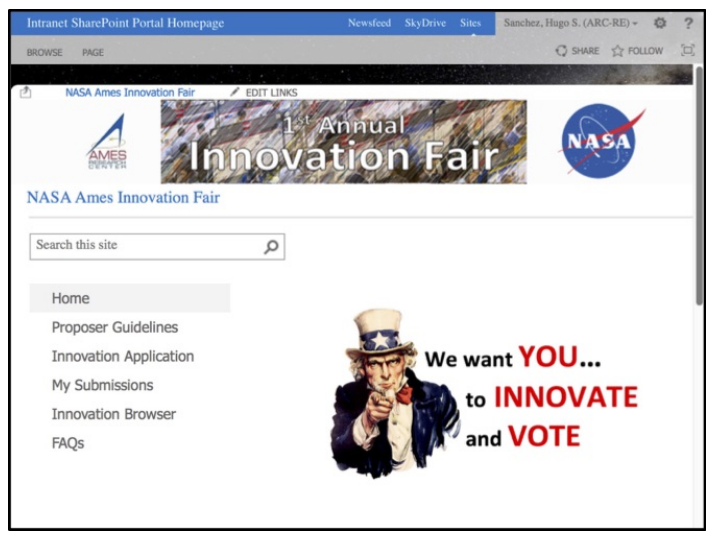

Figure 1. Screenshot of the Innovation Fair homepage electronic proposal submission process provided an interal control mechanism to ensure legal compliance in this area.

The application process was designed to be as simple as possible to encourage submission of all innovative concepts regardless of the level of concept maturity. Additionally, these applications were not passed through an initial senior panel, but made publicly available to the voters for full and open exposure of the innovation. The idea was to promote the free exchange of ideas without the barrier of management oversight panels excluding ideas at an early stage. The application included 14 questions; 5 were multiple choice, 7 were short answer, and 2 were for descriptive entries. The simplified application process was directly aimed at addressing the excessive administrative tasks that are common in traditional innovation processes and identified as a barrier to innovation [11]. The descriptive fields "Idea Description", "Why is this innovative?", and "What NASA strategic goal does this innovation address?" were required of the applicant to promote an educated voter base.

Once accepted, the applicant information was posted to the website. This knowledge capture element allowed potential collaborators and funding sources to search innovation concepts, before, during and after the day of the Innovation Fair. This visibility opened new opportunities for proposers long after the physical event. Each concept is also connected to a discussion page on Sharepoint where peers and concept proposers could exchange thoughts. This site was used by the ARC community to collaborate before, during and months after the physical event.

A customized voting application was developed and implemented on the website (see Section 3, Voting Methodology).

\section{Physical Component}

The physical event was the primary focus of the Innovation Fair, and it allowed face-to-face interactions between proposers and attendants which led to dynamic conversations in a way that was not possible online.

The fair was arranged outdoors in a poster session format, and was collocated with an ARC employee morale event. (Fig 2). Each proposer created a poster board to help pitch their

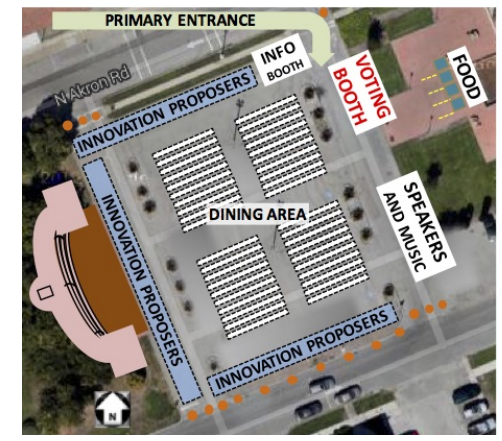

Figure 2. Map of the Innovation Fair Tents co-located with an annual employee morale event.

concept, but no other demonstration technology, physical or virtual was allowed. This was to ensure a level playing field between technical and non-technical innovations. The proposers were arranged in a pseudo-random order which resulted in an even distribution of technical and non-technical concepts around the poster display area. 
Since the Innovation Fair awards would be determined by democratic vote, it was recognized early that marketing and location would impact fair attendance and participation. Thus, the Innovation Fair was strategically collocated with an employee morale event, which provided awareness and attendance for this event. This enabled a larger number of the ARC community to familiarize themselves with the Fair and become voters for innovations that they thought would advance ARC and NASA's future. Finally, during the event, multiple senior managers spoke and encouraged attendants to participate in the Innovation Fair activities.

Due to the relatively high number of proposers (49) and short event duration ( 2 hours), proposers had to be concise with their concept description as voters moved from proposer to proposer. These "elevator speeches" in combination with the diverse group of attendees was designed to foster crossorganizational collaboration aligned with the formal objectives of the Innovation Fair.

\section{Voting Methodology}

One of the key tenets of the Innovation Fair was to provide employees the opportunity to vote on innovations and help shape the future of ARC. In order to meet this tenet the organizers considered the following issuses:

- Possible manipulation of the voting system

- Individuals winning on the merit of personality more than the merit of the innovation

- Risk that an innovation from a particular organization wins solely due to high voter turnout by their organization

The goal was to address all of these concerns while creating a voting system that was simple, transparent and feasible within the physical and virtual environments that were chosen.

In an effort to promote voting based on the merit of the innovation and mitigate the risk of voters teaming up to vote for one specific innovation by their organization, the decision was made to give each voter ten votes with a caveat. A voter could only allocate up to five votes to any given innovation. This meant that a voter could submit one vote for up to a maximum of ten innovations or submit five votes to a minimum of two innovations. The result of this system gave voters i) the flexibility to distribute votes freely between projects based on the perceived merit of the innovation and ii) the opportunity to vote for innovations outside of their organization. In order to address voter manipulation, internal controls are built into an electronic voting application specific to the Innovation Fair voting methodology. Each user voted online using their NASA credentials in accordance with the voting guidance that was provided. This ensured that the internal controls established produced voting results that were reliable. Finally, it was determined that a twelve hour voting window on the day of the fair would be most appropriate to encourage fair attendance and quickly provide voting results to the Center Director and ARC community.

It is important to note that this system still could provide an advantage to popular projects and/or those that are less technically advanced or strategic, but this was determined to be an acceptable outcome because the idea behind seed funding is to fund many ideas that are not fully developed. Additionally, an objective of the Innovation Fair was to experiment with methodologies that would change the way innovative ideas were funded at ARC.

\section{Award Methodology}

After discussion with senior management at the center, the Innovation Fair was allocated $\$ 150 \mathrm{k}$ in innovation award funding. Similar to the voting methodology, it was necessary to develop a award methodology that was simple, transparent, feasible and aligned with the objectives for the Innovation Fair. Thus, many issues needed to be considered:

- Identification of an award structure that incentivized innovators, from both technical and non-technical support organizations

- Determine whether to award multiple innovations or only the top innovation

- Determine optimal funding level for all awards, e.g. each winner receives the same amount of funding or the most popular innovation receives a greater proportion of the funding

- Ensure that the award structure is perceived as fair by Ames employees

As the team researched different award methodologies from Section 2 it became clear that some flexibility would be required to meet the above requirements. Since this was a first of its kind event at $\mathrm{ARC}$, true interest from proposers and fair attendees would not be predictable. Thus, flexibility would allow adaptability to different voting results scenarios that could result from this uncertainty of employee participation as well as the possible risk of disparity between non-technical and technical innovations. This disparity was recognized as a concern early on because the existing ARC culture primarily promotes innovation in technical organizations. Therefore, in the call to proposers, employees were allowed to request up to $\$ 25 \mathrm{k}$ in funding, and it was stated that both technical and non-technical innovations would be funded. This system guaranteed that a minimum of six innovations would receive award funding including at least one non-technical innovation. This methodology provided appropriate incentives for employees to bring forth their innovations and provided additional flexibility to adjust award amounts after analyzing the voting results.

After the voting results were processed by the Innovation Fair team, the top six innovations were submitted as a recommendation to the center director. After review of the results the center director would approve the top six winners to receive the requested funding award.

\section{Success Metrics}

Several metrics were established to measure the success of the Innovation Fair with respect to the formal objectives (Section 1). The metrics focused on quantitative and qualitative feedback from two groups: the innovators and fair attendees. For quantitative measurement, both innovators and fair attendees were given a survey that requested a rating, on several statements, ranging from "1-Strongly Disagree" to "5Strongly Agree":

$$
\begin{array}{ccccc}
\text { Strongly Disagree } & \text { Disagree } & \text { Neutral } & \text { Agree } & \text { Strongly Agree } \\
1 & 2 & 3 & 4 & 5
\end{array}
$$

The innovators were asked to rate their level of agreement with three statements. These statements intended to assess the objectives of collaboration and participation of all ARC organizations from the innovator's perspective.

- I feel that the Innovation Fair fostered collaboration.

- I feel that the Innovation Fair fostered collaboration between different organizations. 
- I feel that the Innovation Fair promoted innovation in mission support (non-technical) organizations.

The fair attendees were asked to complete a separate survey in conjunction with submitting votes for innovations (vote submission was contingent on survey completion) and asked to rate their level of agreement (see scale above) with five statements. These five statements intended to assess the perceived value of the Innovation Fair to attendees, the perceived ability to influence investment decisions at the center, and the perception of contribution to the innovation culture at ARC.

- I want to propose at next year's Innovation Fair.

- I want to attend next year's Innovation Fair.

- I learned about new innovations at ARC.

- I feel my vote will influence the investement decisions at the center.

- I feel more empowered to innovate after attending the Innovation Fair.

The Innovation Fair team also collected informal qualitative feedback from fair attendees, voters, proposers, managers and senior managers over the following weeks to gauge the success of the fair and gather input to improve future iterations of the Innovation Fair.

\section{RESULTS}

The First Annual Ames Innovation Fair was held on March 3rd, 2016 at NASA Ames Research Center (Fig. 3).

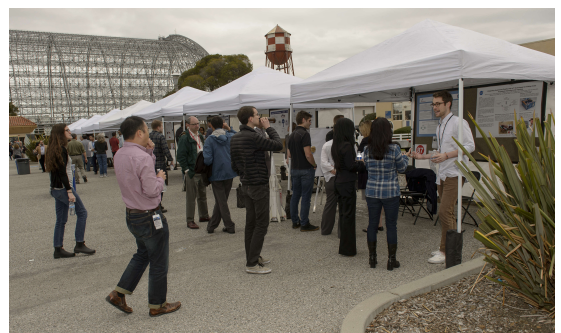

Figure 3. Attendees of the 1st Annual Ames Innovation Fair

Fair attendees collaborated with innovators (Fig. 4) and cast their votes for what they viewed as the most innovative concepts.

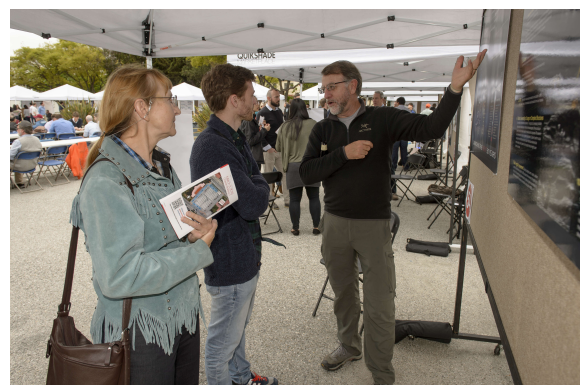

Figure 4. An Innovator pitches his innovation to Fair attendees

This event was also supported and attended by ARC senior management (Fig. 5).

The following sections discuss the innovator participation

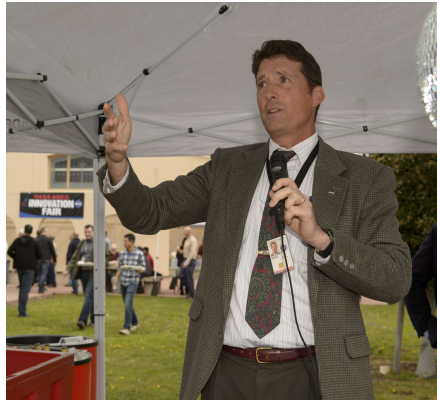

Figure 5. Deputy Center Director, Tom Edwards, speaks to fair attendees

survey results, general participation survey results, and the voting results.

\section{Innovator Participation Survey Results}

A total of 49 civil servant-led innovation proposals were showcased at the Innovation Fair. The distribution by total percentage of innovators and voters by directorate can be seen in Fig. 6, where innovators are represented by the blue bar. Ames organizational designations can be found in Table 1.

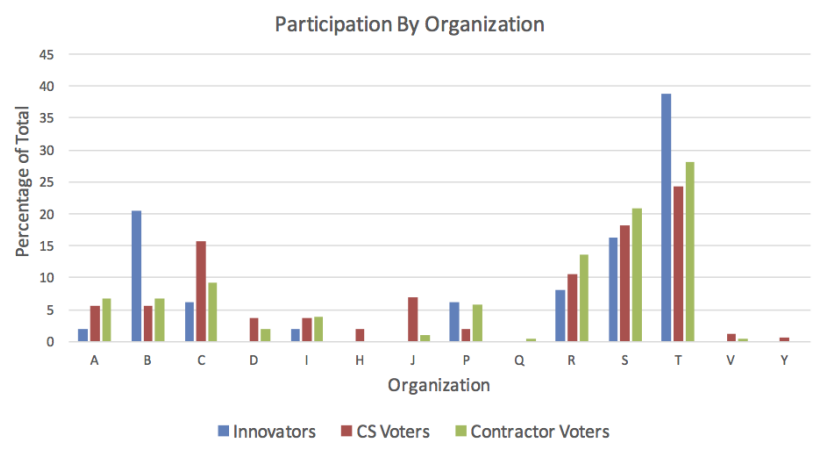

Figure 6. Participation by organization

Table 1. NASA ARC Organizations

$\begin{array}{cl}\text { A } & \text { Aeronautics } \\ \text { B } & \text { Partnerships } \\ \text { C } & \text { Finance } \\ \text { D } & \text { Administration } \\ \text { I } & \text { Information Technology } \\ \text { H } & \text { Human Capital } \\ \text { J } & \text { Center Operations } \\ \text { P } & \text { Programs and Projects } \\ \text { Q } & \text { Quality \& Mission Assurance } \\ \text { R } & \text { Engineering } \\ \text { S } & \text { Science } \\ \text { T } & \text { Exploration Technology } \\ \text { V } & \text { Strategic Communications \& Education }\end{array}$

One of the objectives of the Innovation Fair was changing the traditional focus of innovation on technical advancement to a culture that includes non-technical organizations. The results show that innovators came from traditional technical organizations, such as aeronautics, space technology, engineering, etc. and from non-technical organizations, such as finance and partnerships. As part of the survey to innovators after the event, they were specifically asked if they felt that the Innovation Fair promoted innovation in mission support 
organizations, and there was overall agreement with this statement (Fig. 7). This data indicates that mission support organizations have innovative ideas that can contribute to the success of ARC when given a platform from which to showcase their ideas.

In Fig. 7, the results of the innovator survey are plotted. The innovator survey questions that focused on the fair objective of collaboration showed that there was agreement that the Innovation fostered collaboration between different organizations. Other informal feedback was collected, and an email was received that provided evidence of collaborative opportunities that were generated because of the Innovation Fair:

\section{Just wanted to follow-up from our brief chat this afternoon to provide you with a very short testimonial on how the innovation fair inspired future collaborations and exploration of alternate venues in pursuit of the idea brought forth at the fair...}

Both the quantitative and qualitative feedback that was received suggests that the Innovation Fair was successful in meeting the objective of providing a forum for collaborative opportunities to develop.

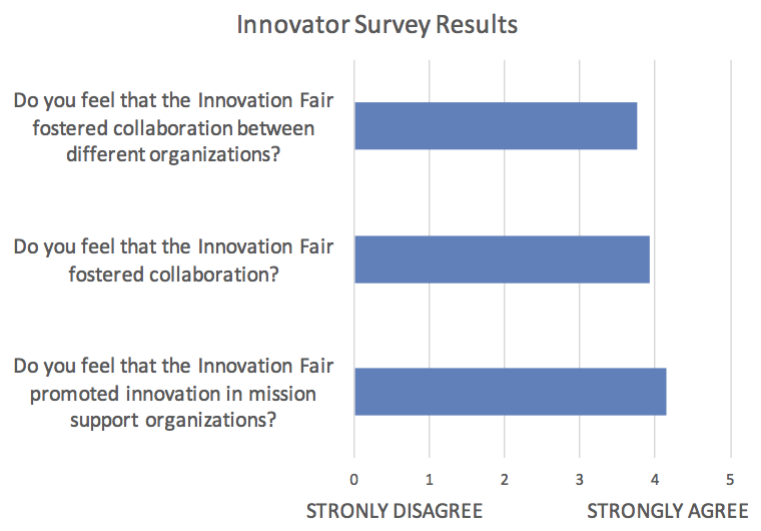

Figure 7. Innovator Survey Results

\section{General Participation Survey Results}

Voter participation in the Innovation Fair was approximately $20 \%$ of the Ames community. Considering other events at ARC with voluntary attendance typically draw from $1 \%$ to $10 \%$ attendance, $20 \%$ is relatively high, especially for a first of its kind event. Throughout the two hour event, many proposers were engaged with the ARC community for a majority of the time, and there were often crowds of people surrounding individual booths. While it is challenging to assess the successful turnout with an arbitrary percentage of attendance number, feedback from voters and proposers suggests that higher level of turnout would require considering extending the presentation window or other fair format modifications to accommodate additional traffic.

Figure 6 shows the distribution of voters based on organization. The results show that the organizations where innovation is already part of the culture, participation as a voter and innovator was relatively high, e.g Exploration Technology $(\mathrm{T})$, Science (S), and Engineering (R). However, voter and innovator participation by some technical and most nontechnical organizations was low. Participation as a voter and innovator by Partnerships (B), Finance (C), and Programs and Projects $(\mathrm{P})$ does show some increased interest as compared to the organizations where participation was very low (Code $\mathrm{A}, \mathrm{D}, \mathrm{J}, \mathrm{H}, \mathrm{I}$, and Q). The low participation organizations suggest that innovation culture barriers still exist and events such as the Innovation Fair can serve as a catalyst for the transformation to an innovation culture over time.

A short survey was also included during the voting process. The survey results collected from voters were generally positive as can be seen in Fig. 8. The voters strongly felt that they learned about the work their colleagues are pursuing and that the event was worth attending again in the future, indicating the the social interaction and sense of community that the Innovation Fair offered was aligned with the collaborative objectives of the fair. Voters also agreed that they felt empowered to innovate and that they felt their votes would be influential. This suggests that the ARC community felt they could help shape the future of ARC, which directly aligns with one of the goals of the Innovation Fair. The voters empowerment to innovate can be seen as a dynamic of cultural change and indicates that the fair was a step in the direction of positively influencing the innovation culture at ARC.

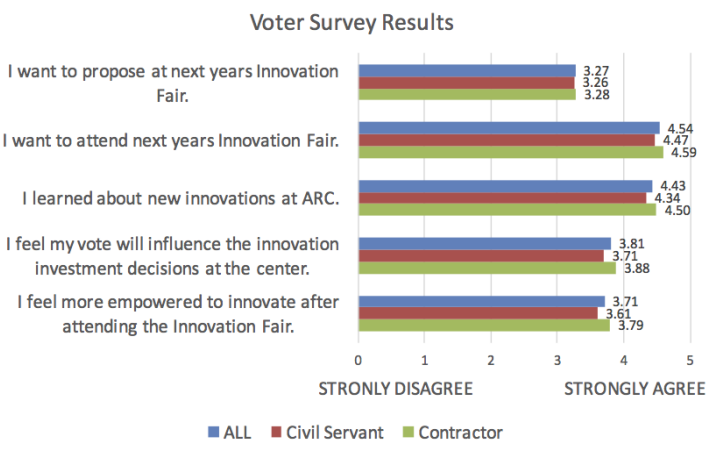

Figure 8. Ames Innovation Fair Survey Results

In general, the voter responses indicate that the event met the goals to promote an innovation culture, foster innovation across organizations, and increase employee influence on NASA's future. However, further study needs to be done in the future that assesses the cultural impact of the fair over a longer time horizon.

\section{Voting Results}

The voting data was analyzed to identify the top six innovations, the proportion of voters that applied their votes to projects outside of their home organization, and the level of potential cross-organizational collaboration that resulted from the physical component of the Fair.

The results of the voting can be found in Table 2 and include five parameters:

Votes the sum of the number of votes applied to each project by each voter.

Organization the organization that the innovation originated from, identified as a letter designation (see Table 1 for full organization names)

$\%$ Vote Cross-Org the percentage of votes that were given by voters from an organization different than the organization the innovation originated from 
Table 2. Innovation Fair voting results for all 49 innovations.

\begin{tabular}{|c|c|c|c|c|c|}
\hline Innovations & Votes & Organization (Org) & \% Vote Cross-Org & Collaboration Interest & \% Cross-Org Interest \\
\hline Large Diffractive Aperture for NEA Detection & 113.00 & S & $57 \%$ & 20 & $60 \%$ \\
\hline Project Planning and Control System & 106.90 & $\mathrm{C}$ & $31 \%$ & 15 & $27 \%$ \\
\hline EColl: Escape * Collaborate * Innovate & 92.67 & T & $70 \%$ & 26 & $85 \%$ \\
\hline Inside Ames 2.0 & 68.58 & $\mathrm{R}$ & $91 \%$ & 13 & $77 \%$ \\
\hline NASA Mirco/NanoSatellite Cost Model & 68.16 & $\mathrm{C}$ & $21 \%$ & 11 & $45 \%$ \\
\hline ADEPT-based Active Lift Guided Planetary Entry & 62.67 & T & $47 \%$ & 15 & $60 \%$ \\
\hline MiDAR UV - Multispectral Detection and Active Refl & 61.33 & $\mathrm{~S}$ & $27 \%$ & 12 & $25 \%$ \\
\hline Seeker: Vision-Based Nanosat Proximity Operations & 42.63 & $\mathrm{R}$ & $52 \%$ & 12 & $67 \%$ \\
\hline Drill-Integrated Neutron/Gamma Ray Spectrometer & 38.00 & S & $55 \%$ & 3 & $67 \%$ \\
\hline Ames Collaboratory & 36.71 & B & $84 \%$ & 13 & $92 \%$ \\
\hline CNT development for medical diagnostic sensing & 36.22 & T & $58 \%$ & 7 & $29 \%$ \\
\hline Augmenting Arcjet Heat Flux with Diode Array Lamps & 36.17 & $\mathrm{P}$ & $81 \%$ & 5 & $100 \%$ \\
\hline Database of Ames Personnel Technical Capabilities & 36.00 & B & $58 \%$ & 8 & $63 \%$ \\
\hline Oculometric Assessment of Neural Health & 32.33 & T & $41 \%$ & 10 & $50 \%$ \\
\hline IT solution deployment for Next Generation Zero & 31.39 & T & $56 \%$ & 7 & $43 \%$ \\
\hline In-flight Safety Assistant & 30.78 & T & $64 \%$ & 6 & $33 \%$ \\
\hline Accelerating Solutions for Earth and Space & 29.20 & B & $53 \%$ & 14 & $79 \%$ \\
\hline Apollo 50-Launching a New Era of Lunar Exploration & 29.00 & B & $83 \%$ & 7 & $57 \%$ \\
\hline Make It There - Don't Take It There & 28.62 & B & $80 \%$ & 6 & $83 \%$ \\
\hline Self-Repairing Robots & 26.36 & $\mathrm{~T}$ & $32 \%$ & 5 & $60 \%$ \\
\hline Prediction Market Crowd Sourcing (PMCS) & 24.00 & $\mathrm{~S}$ & $38 \%$ & 6 & $17 \%$ \\
\hline Automatic Tracking of Timeline Execution for Crew & 29.00 & $\mathrm{~T}$ & $62 \%$ & 9 & $11 \%$ \\
\hline Teaching robot collectives & 24.29 & $\mathrm{~T}$ & $63 \%$ & 8 & $25 \%$ \\
\hline $100 \mathrm{~kg}$ Options for Lunar Development & 20.53 & B & $58 \%$ & 8 & $63 \%$ \\
\hline Machine Learning to Predict Amazon Future & 20.00 & $\mathrm{~T}$ & $30 \%$ & 3 & $33 \%$ \\
\hline Ames' Vision Deployment using Systems Thinking & 20.00 & $\mathrm{~T}$ & $40 \%$ & 4 & $75 \%$ \\
\hline Beam Me Up! & 18.33 & $\mathrm{P}$ & $89 \%$ & 6 & $100 \%$ \\
\hline Life and biosignature detection with MinION & 17.00 & $\mathrm{~S}$ & $24 \%$ & 10 & $40 \%$ \\
\hline Physiological-Measure Tool Suite & 17.00 & A & $29 \%$ & 4 & $100 \%$ \\
\hline Automated Free Flying Commercial Microgravity Lab & 16.40 & B & $72 \%$ & 8 & $88 \%$ \\
\hline Addressing Climate Change Challenges through On Or & 16.00 & B & $94 \%$ & 3 & $67 \%$ \\
\hline Detection of Organics with out sample handling & 15.00 & $\mathrm{~S}$ & $53 \%$ & 9 & $22 \%$ \\
\hline Self-Healing Space Electronics System & 15.00 & $\mathrm{~T}$ & $67 \%$ & 4 & $50 \%$ \\
\hline Single Event Upset Counter & 14.00 & T & $36 \%$ & 2 & $0 \%$ \\
\hline Artificlal Life: a tool for Origin Studies & 13.00 & $\mathrm{~T}$ & $23 \%$ & 2 & $50 \%$ \\
\hline Intelligent Systems for NASA's Big Data Problems & 13.00 & $\mathrm{~T}$ & $15 \%$ & 7 & $14 \%$ \\
\hline Flights of Genius & 12.71 & B & $39 \%$ & 3 & $0 \%$ \\
\hline Augmented Reality for Aiding Procedure Execution & 14.00 & T & $50 \%$ & 14 & $29 \%$ \\
\hline Tunable materials with bio-assisted manufacture & 10.71 & $\mathrm{~S}$ & $25 \%$ & 4 & $25 \%$ \\
\hline Implementation of Continuous Web Application Monit & 9.61 & 1 & $31 \%$ & 5 & $20 \%$ \\
\hline Automatic classification of transit-like signals & 9.00 & $\mathrm{R}$ & $100 \%$ & 3 & $100 \%$ \\
\hline A Physically Unclonable Function based on a CNT & 8.29 & T & $28 \%$ & 3 & $0 \%$ \\
\hline Planetary Protection for Icy Bodies & 8.00 & $\mathrm{~S}$ & $75 \%$ & 4 & $25 \%$ \\
\hline Mission to America - CMAPP & 6.22 & B & $52 \%$ & 3 & $33 \%$ \\
\hline Distributed Optical Receiver Apertures & 6.00 & $\mathrm{R}$ & $83 \%$ & 3 & $33 \%$ \\
\hline OmniProp & 5.00 & $\mathrm{P}$ & $80 \%$ & 1 & $100 \%$ \\
\hline Plasma Channel Switch with sub-10 Volts operation & 4.82 & T & $38 \%$ & 1 & $0 \%$ \\
\hline A Self-Healing Process against Radiation Hardening & 4.00 & $\mathrm{~T}$ & $50 \%$ & 3 & $0 \%$ \\
\hline
\end{tabular}

Collaboration Interest the number of voters that identified an interest in collaborating with the innovator of the favored innovation

$\%$ Cross-Org Interest the percentage of those voters interested in collaborating with the innovator that are not from the organization that the innovation originated from

The voting results were consistent with the intent of the voting methodology, which was to create a fair process that encouraged voters to look at all innovations and not just those from within their own organizations. On average, $53 \%$ of votes came from organizations outside of the innovator's home organization. One winning concept, Inside Ames 2.0 , received $91 \%$ of votes from other organizations. This data suggests that Innovation Fair participants were willing to treat the event as an opportunity to collaborate across organizational lines, and competition between innovators did not seem to be an issue that inhibited cross-organizational collaborative opportunities. Furthermore, thirty of the innovations received more than $50 \%$ of their votes from voters that did not come from their home organizations. Additionally, 44 innovations had voters not from the innovator's home organization interested in collaborating with the innovator. This implies that a segment of voters within the Innovation Fair structure were engaged in innovations that were not within their own organization. These results provide evidence that 'innovation contest methods' can foster cross-organizational collaboration, providing innovators with a diverse knowledge base to help advance their innovations.

In Table 3, the top seven winning innovations are listed by organizational type. It is significant to note that participants voted for both non-technical and technical innovations with an almost even split among the top seven. This suggests that the structure of the Innovation Fair created an environment that showcased non-technical innovations on an even level with technical innovations. Additionally, the flexibility that was built into the voting/award methodology provided the Center Director ability to include a seventh winner among the list of awardees.

Table 3. Organizational type of the top seven winners.

\begin{tabular}{lc} 
Innovation & Type \\
\hline Large Diffractive Aperture for NEA Detection & Technical \\
\hline Project Planning and Control System & Non-Technical \\
\hline EColI: Escape * Collaborate * Innovate & Non-Technical \\
\hline Inside Ames 2.0 & Non-Technical \\
\hline NASA Mirco/NanoSatellite Cost Model & Non-Technical \\
\hline ADEPT-based Active Lift Guided Planetary Entry & Technical \\
\hline Multispectral Detection and Active Reflectance in Ultra-Violet & Technical \\
\hline
\end{tabular}

A description of each winning innovation can be found in the Appendix. 


\section{LESSONS LEARNED}

While an event like the Innovation Fair can catalyze the shift to a more innovative culture at ARC, it is the sustained, year over year effort of the fair that will be an important component of the new innovation culture at ARC. As such, it is important to integrate lessons learned into future iterations of the fair to better achieve objectives and maximize the positive cultural contribution over time.

Several key lessons were learned, but three were identified as most critical for improving future iterations of the Innovation Fair. First, more targeted marketing strategies will be need to promote an innovation culture in some technical and all non-technical organizations. As previously discussed, many non-technical organizations had very low voter turnout for the fair, so more aggressive marketing strategies will need to be devised and implemented. Non-technical participation in the Innovation Fair was one of the three main objectives of the event, and increased year over year participation might be an indicator that the grassroots innovation culture is taking root in non-technical organizations.

Second, virtual collaboration was under-utilized. Online collaboration can be a very important forum for communicating, and devising a strategy for greater utilization of the online platform will be required. As technology for online and virtual collaboration has evolved to the point of downloading an application on a smart phone and engaging in discussion virtually anywhere, additional investigation into new collaboration tools that may be a better fit for the Innovation Fair will likely be needed for every yearly iteration of the fair. This increase in collaboration ties directly to one of the three objectives of the fair, and an increase in virtual collaboration would certainly enhance the innovation culture at ARC.

Finally, it will be important to determine the long term impact of the fair on both the culture of ARC as well as the contribution to mission success. Much of the data that has been presented indicates that the Innovation Fair has, to this point, been successful. However, it must be acknowledged that if the impact on the innovation culture at ARC is not sustained, or new technologies that create or enhance NASA' mission success are not developed, these early signs of success mean little. Skeptical stakeholders are interested in whether or not this new method of allocating award funding will be effective. In truth, the authors also approach this new model with cautious optimism, hoping that successful innovation practices, such as the Innovation Fair, will transfer to the innovative but sometimes very bureaucratic organization that is NASA. While data collected suggests that the seeds of a grassroots innovation culture have taken root at ARC, more time and data will be needed to validate the promising initial results.

\section{Conclusion}

The First Annual NASA Ames Innovation Fair was successful in establishing a peer-driven funding call and forum for technical and non-technical organizations within the center. The enthusiasm generated among the Ames workforce after the First Annual NASA Ames Innovation Fair was best expressed by one of the Fair attendees about innovators pitching their ideas at the fair:

Usually people talk about the work they have already done, but it was inspiring to learn about what they want to do!
Based on the feedback, survey results, and voting results, the Innovation Fair concept was effective in meeting the objectives outlined to promote an innovation culture at ARC. The Innovation Fair implementation provided an environment that promoted employee engagement in the future of ARC by involving them directly in the decision-making process. Attendees had a largely positive experience when engaging in discussion with innovators about projects that influence the future of ARC. Additionally, attendees utilized their role as a voter in the grassroots selection of projects, that are innovative and meet NASA's strategic goals, resulting in seven innovative ideas being awarded funds to grow their innovations. Finally, there was a significant amount of crossorganizational collaboration and interest among the attendees and innovators. Thus, beginning the process of breaking down organizational silos within NASA Ames. Since the completion of the Innovation Fair, the Agency has bench marked the employee voting approach for innovation selection as a model for the NASA Innovation KickStarter (NIKS) program, which was released across the 10 NASA centers at the Agency Innovation Mission Day on November 1, 2016.

The Innovation Fair took a little over a year to implement with small investments from NASA Ames. Although the return on this investment will not be fully known in the immediate future, the results show that the culture is moving in the right direction. Sustained support by NASA Ames center management into the future will allow the team to build upon the strong foundation of the Innovation Fair concept. Integration of additional knowledge production methods for the Second Annual Innovation Fair will help to increase non-technical organization involvement, increase the quality of cross-organizational collaboration, and maintain a sense of ownership in NASA's future among the workforce. Achieving these goals and sustaining these results will lead to a powerful culture of innovation for the advancement of NASA's missions in aerospace.

\section{APPENDICES}

\section{Large Diffractive Aperture for NEA Detection}

NEAscope is a mission concept utilizing a large (1-m) diffractive optical system, stowable and deployable from a small satellite or cubesat platform, and designed to detect Near Earth astreroids (NEAs). If funded, the proposed work will focus on developing the diffractive optical element (DOE) for NEAscope. The major challenge of space observatories is the limited size of optics. In addition to being heavy themselves, traditional optics also require large volumes of coolant for IR observations. To resolve these limitations, the aim of this work is to deliver a proof-of-concept for a lightweight, low volume, deployable film, primary optic for small spacecraft. The proposed study will be comprised of computational and optical design work in parallel with laboratory validation. The design and fabrication of the aperture will be completed by Co-I Milster's group at University of Arizona's Optical Science Center. This group will iterate on designs for a phased optical array. Scaled versions of these optics will be delivered to NASA ARC for performance testing to verify required parameters for detecting small NEAs at IR wavelengths.

\section{Project Planning and Control System}

To better control, manage and plan mission budget, we propose to design and develop an Excel-based reporting system 
to extract and integrate plan, actual and transaction data at the projects' lowest Work Breakdown Structure (WBS) levels, perform computations, and generate dynamic reports. The system utilizes programming, efficient formulas, and connection with SAP BOBj. This system will streamline the budgeting process by helping users to create project and phasing plans, and will significantly reduce time devoted to data research and analysis (from hours to minutes in many cases). As a result, this tool will free up analyst time to gain insights into project performance and help managers make key decisions tracking status and variances to plan.

\section{EColI: Escape * Collaborate * Innovate}

We are building a place where the Ames workforce can escape to focus and think openly; we are building a place where the Ames workforce can collaborate with familiar and unfamiliar colleagues; we are building a place where the Ames workforce can innovate! This will be the place NASA should be - a place that offers a variety of environments to suit your dynamic needs and promote productivity, creativity and engagement, pulling from the latest research in social psychology (including just the right ambient noise, furniture selection, colors and hint of nature) infused with technology.

\section{Inside Ames 2.0}

Inside Ames 2.0 will replace the current Inside Ames website as a new, up-to-date internal website. It will bring together the multitude of services, resources, and webpages within the NASA Ames infrastructure, and make it accessible from a single user-friendly, accessible, and functional website. A major problem with the current information infrastructure at NASA Ames is that although resources are available, they are difficult to find and not effectively communicated to the workforce. As such, new and existing initiatives and resources remain unknown and underutilized. The same inefficiencies in information infrastructure make collaboration and general awareness within the Center difficult, and hinder the growth of a strong Ames community, particularly when it comes to new hires. Inside Ames 2.0 will address these issues with user-friendly database search tools that allow employees to easily navigate Ames' organizational structure and find org websites, find relevant resources, services, and facilities, and learn about other projects and people within the center. Inside Ames 2.0 also has the potential to serve other important functions, such as distributing news and recent publications, and connecting hiring opportunities with internal talent.

\section{NASA Mirco/NanoSatellite Cost Model}

Cost modeling tool that uses technical engineering and operating space environment data to generate cost estimate for development of NASA Micro/NanoSatellites Cost Tool based on historical data and complexity factors. The tool outputs development cost for all NASA Work Breakdown Structure (WBS) and for Phase B-D.

\section{ADEPT-based Active Lift Guided Planetary Entry}

This project is to develop an integrated guidance and control $(\mathrm{G \& C})$ architecture for large payload delivery to Mars (and beyond) by investigating the feasibility of using moving mass control technology as a means of G\&C effector for ADEPT configured planetary vehicles. An internal moving mass actuator control systems provides an active control mechanism for re-entry vehicles without direct interaction with the flow filed of the atmosphere. A scaled ADEPT hardware prototype will be developed to demonstrate, through extensive bench/flight tests, the effectiveness of the proposed moving mass actuation concept for modulating the flight path angle, to achieve precision guidance control for planetary Aerocapture and Entry, Descent and Landing (EDL). The revolutionary aspect of this project is that such integrated G\&C methods for ADEPT configured re-entry vehicles have never been demonstrated beyond conceptualization. Therefore, this project offers a unique opportunity for developing and validating the scaled hardware prototype as well as the advanced guidance and control algorithm.

\section{Multispectral Detection and Active Reflectance in Ultra- Violet}

We present a novel remote sensing technique and instrument for Multispectral Imaging, Detection and Active Reflectance in Ultra-Violet (MiDAR UV) with next-generation scientific capabilities for simultaneous in-situ, airborne and spaceborne measurements and optical communications. MiDAR consists of an active optical transmitter and passive receiver in monostatic and bistatic configurations. The MiDAR transmitter emits coded narrowband structured illumination to generate high-frame-rate multispectral video, perform realtime radiometric calibration and provide a high-bandwidth simplex optical data-link under a range of ambient irradiance conditions, including darkness. Multipurpose sensors, such as MiDAR, which fuse active sensing and communications capabilities, may be particularly well-suited for mass-limited robotic exploration of Earth and the solar system and represent a possible new generation of instruments for active optical remote sensing.

\section{ACKNOWLEDGMENTS}

The authors would like to thank NASA Ames Research Center Director Eugene Tu and Chief Information Officer Jerry Davis for their guidance and support in making the Innovation Fair a reality. The authors would also like to thank NASA's Convergent Aeronautics Solutions project for funding the Fair. Also, a special thanks to NASA Logisitics Lead Darlene Gross for her engaging support and to the NASA FIRST program.

\section{REFERENCES}

[1] C. Franzoni, H. Sauermann, "Crowd science: The organization of scientific research in open collaborative projects", Research Policy, vol. 43, pp. 1-20, 2014.

[2] Centennial Challenges Web site: http://www.nasa.gov/directorates/spacetech/centennial_challenges/.

[3] Center Innovation Fund Web site: http://www.nasa.gov/directorates/spacetech/innovation_fund/index.html.

[4] NASA Innovative Advanced Concepts (NIAC) Web site: http://www.nasa.gov/directorates/spacetech/niac/index.html.

[5] Space Technology Research Grants Web site: http://www.nasa.gov/directorates/spacetech/strg/index.html.

[6] Game Changing Development Program Web site: http://www.nasa.gov/directorates/spacetech/game_changing_development/index.html.

[7] Technology Demonstration Missions Web site: 
http://www.nasa.gov/mission_pages/tdm/main/index.html.

[8] NARI Seedling Fund Web site: http://nari.arc.nasa.gov/seedling.

[9] Transformative Aeronautics Concepts Program Web site: http://www.aeronautics.nasa.gov/programs-tacp.htm.

[10] NASA Innovation

Pavillion Web site: http://www.nasa.gov/offices/COECI/platforms/innocentive.html.

[11] Waszak, M., Agency Barriers to Innovation, White paper presentation, February 2013, https://www.nasa.gov/sites/default/files/files/KThompson_BarrierstoInnovation.pdf.

[12] Microsoft SharePoint Web site: https://support.office.com/en-us/article/What-is-SharePoint-97b915e6-65 1b-43b2-827d-fb25777f446f.

[13] R. Merton, The Sociology of Science: Theoretical and Empirical Investigations, University of Chicago Press, 1973.

[14] Innocentive Web site: https://www.innocentive.com/about-us/.

[15] M. Nielson, Reinventing Discovery: The New Era of Networked Science, Princeton University Press, 2011.

[16] S. Cooper, F. Khatib, A. Treuille, J. Barbero, J. Lee, M. Beenen, A. Fay-Leaver, D. Baker, Z. Popovic, "Predicting protein structures with a multiplayer online game", Nature, vol. 466, no. 7307, pp. 756-760, 2010.

\section{BIOGRAPHY}

Dr. Sarah D'Souza is an Aerospace Flight Systems Engineer at NASA Ames

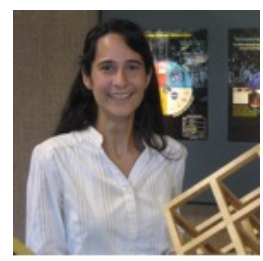
Research Center in the Systems Analysis Office. She is currently applying her expertise in vehicle trajectory modeling and simulation to advance NASA's mission to build a UAV Traffic Management System that enables safe and autonomous operation of UAVs in the national airspace. Dr. D'Souza received her Bachelor's of Science (2005), Master's Degree (2009), and Doctorate (2013) in Mechanical and Aerospace Engineering at the University of California at Davis.

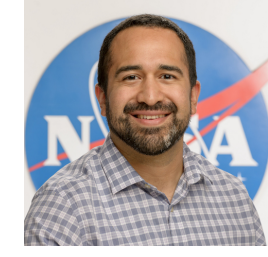

Hugo Sanchez received a B.A. in Physics from U.C. Berkeley in 2008. He has been with NASA Ames Research Center for 9 years. A member of Systems Engineering Branch, he has served as Spacecraft Bus Systems Engineer for the Edison Demonstration of Smallsat Networks (EDSN) and BioSentinel projects. Prior to EDSN, he was a proposal lead for new concepts in ARCs Mission Design Center. His technical focus was on radio communication, data management, and trajectory planning.

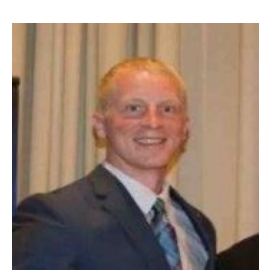

Ryan Lewis grew up in the suburbs around Milwaukee, WI and joined the Navy shortly after 9/11/2001. After spending five years stationed in San Diego as an avionics technician and completing a deployment on the aircraft carrier USS John C. Stennis, he returned to Milwaukee where he finished his BBA in Accounting at the University of Wisconsin - Milwaukee. After briefly working as an accountant, he returned to California where he completed his MBA at Pepperdine University. He joined Ames Research Center in 2013 as a budget analyst for the IT directorate. Currently, Ryan lives in Houston, TX, and is a program analyst for the Orion Multi-Purpose Crew Vehicle program at Johnson Space Center. 